\title{
Insight into the pathogensis of polycystic ovarian syndrome
}

\author{
Yong Wook Jung ${ }^{1 \oplus}$, Gun Ho Lee ${ }^{2 \oplus}$, You Jung Han ${ }^{1}$, and Dong Hyun $\mathrm{Cha}^{1} * \oplus$ \\ ${ }^{1}$ Department of Obstetrics and Gynecology, CHA Gangnam Medical Center, CHA University College of Medicine, Seoul, Korea \\ ${ }^{2}$ Department of Obstetrics and Gynecology, CHA Gumi Medical Center, CHA University College of Medicine, Gumi, Korea
}

\begin{abstract}
Polycystic ovarian syndrome (PCOS) is the most common endocrine disorder in women, which is characterized by the oligo/ anovulation, hyperandrogenism (HA) and polycystic ovarian morphology which are diagnostic criteria. PCOS has diverse clinical aspects in addition to those diagnostic criteria including increased risk for cardiovascular diseases, metabolic syndrome, dyslipidemia, type 2 diabetes and impaired fertility. Because of the heterogeneity of the disease, the pathogenesis of the disease has not been elucidated yet. Therefore, there is no cure for the endocrinopathy. HA and insulin resistance (IR) has been considered two major pillars of the pathogenesis of PCOS. Recent advances in animal studies revealed the critical role of neuroendocrine abnormalities in developing PCOS. Several pathways related to neuroendocrine origin have been investigated such as hypothalamus pituitary ovarian axis, hypothalamus pituitary adrenal axis and hypothalamus pituitary adipose axis. This review summarizes the current knowledge about the role of HA and IR in developing PCOS. In addition, we review the results of recent genome wide association studies for PCOS. This new perspective improves our understanding of the role of neuroendocrine origins in PCOS and suggest a novel potential therapeutic target for the treatment of PCOS.
\end{abstract}

Key words: Polycystic ovary syndrome, Genome-wide association study, Genetics, Hyperandrogenism, Insulin resistance.

\section{Introduction}

Polycystic ovarian syndrome (PCOS) is a complex endocrine disorder which is characterized by the oligo/anovulation, hyperandrogenism (HA) and polycystic ovarian morphology (PCOM). This endocrinopathy affects approximately $5 \%$ to $20 \%$ of women in their reproductive age [1]. It is associated with various reproductive, endocrine, and metabolic disorders such as infertility, dyslipidemia, metabolic syndrome, type 2 diabetes and cardiovascular disease $[2,3]$.

PCOS was first identified by Irving Stein and Michael Leventhal as the combination of hirsutism, chronic anovulation and obesity in 1935 [4]. Because of heterogeneous phenotypes of PCOS, a confirmative clinical definition of the disease has not been determined yet. The diagnostic criteria were redefined at a National Institute of Health (NIH) conference in 1990 [5]. The $\mathrm{NIH}$ criteria included dysovulation (oligo/anovulation) associated with clinical and/or biological HA after exclusion of disorders that mimic hyperandrogenemia, regardless of ultrasound features suggestive of a PCOM. Since then, the American Society for Reproductive Medicine (ASRM) and the European Society of Human Reproduction and Embryology (ESHRE) developed the Rotterdam consensus criteria in 2003 and the Androgen excess PCOS Society (AE-PCOS) decided HA to be a mandatory

Received: 19 May 2020, Revised: 7 June 2020, Accepted: 7 June 2020, Published: 30 June 2020

*Corresponding author: Dong Hyun Cha, Ph.D. (D) https://orcid.org/0000-0003-0722-1714

Department of Obstetrics and Gynecology, CHA Gangnam Medical Center, CHA University, 566 Nonhyeon-ro, Gangnam-gu, Seoul 06135, Korea.

Tel: +82-2-3468-3000, Fax: +82-2-3468-2638, E-mail: chadh001@chamc.co.kr

Conflict of interest: The authors declare that they do not have any conflicts of interest.

(C) This is an open-access article distributed under the terms of the Creative Commons Attribution Non-Commercial License (http://creativecommons.org/licenses/by-nc/4.0/) which permits unrestricted non-commercial use, distribution, and reproduction in any medium, provided the original work is properly cited.

(c) Copyright 2020 by the Korean Society of Medical Genetics and Genomics 
criterion for the diagnosis of PCOS. An international evidencebased guideline for the assessment and management of PCOS announced in July 2018. The evidence-based guideline recommends the use of the Rotterdam diagnostic criteria requiring both $\mathrm{HA}$ and irregular cycles in adult and do not recommend ultrasound in adolescents due to overlap with normal reproductive physiology [6]. The patient must present two of the following three PCOS features for a diagnosis: clinical and/or biochemical androgen excess, oligo-ovulation or anovulation, and PCOM on ultrasound $[6,7]$. The ultrasound features of PCOM were as follow: Using vaginal ultrasound transducers with a frequency bandwidth that includes $8 \mathrm{MHz}$, the threshold for PCOM should be on either ovary, a follicle number per ovary of $>20$ and/or an ovarian volume $\geq 10 \mathrm{~mL}$, ensuring no corpora lutea, cysts or dominant follicles are present.

PCOS has various clinical phenotypes in addition to those diagnostic criteria including increased risk for cardiovascular diseases, metabolic syndrome, dyslipidemia, type 2 diabetes and impaired fertility. Even if pregnancy is achieved, women with PCOS have a greater risk of pregnancy related complications such as gestational diabetes, preeclampsia, increased risk of miscarriage and premature delivery $[8,9]$. In addition, outcome of in vitro fertilization (IVF) treatment for patients who diagnosed with infertility and PCOS has been characterized by lower fertilization rate, impaired embryo cleavage and lower implantation rates of embryo, and a higher miscarriage rate even though women with PCOS have an increased number of oocytes [1016].

Multiple factors with genetic and environmental origins affect PCOS development. Because of its heterogenous clinical features and complex multifactorial conditions, the exact pathogenesis of PCOS remains unknown. Potential causes of PCOS have been suggested as following: HA, insulin resistance (IR), high androgen exposure during embryonic period, chronic inflammation, endocrine disorders and genetic factors. Of those, HA and IR consist of the two pillars in the development of PCOS. The pivotal role of HA and IR and their relationship have been extensively reviewed with recent perspectives on the pathogenesis of PCOS in terms of HA, IR and genetics.

\section{Hyperandrogenism}

Hyperandrogenism is the important feature in PCOS. Majority of the patients represents HA. Women with hyperandrogenic PCOS present with increase levels of not only androgen and proandrogen, but also the enzymes that related to produce andro- gen. Androgen has multiple sources, such as ovary, adrenal gland and adipose tissues [17]. Although major source of androgen is the ovary, adrenal gland contributes to androgen production substantially. Several pathways are involved in increasing androgen in PCOS.

First, intrinsic steroidogenic dysregulation in theca cell results in ovarian androgen excess in PCOS [18]. Lutenizing hormone $(\mathrm{LH})$ from pituitary gland binds $\mathrm{LH}$ receptor in theca cell. $\mathrm{LH}$ stimulate adenylate cyclase via G-protein coupled receptor. Adenylate cyclase produce cyclic adenosine monophosphate (CAMP) from adenosine triphosphate. The cAMP activates protein kinase $A$ to stimulate steroidogenesis in theca. Theca cell converts cholesterol into androgen by using CYP11A, CYP17 and $3 \beta$-hydroxysteroid dehydrogenase (HSD) enzyme in response to LH. Then, the androgen produced from theca cell diffuse into granulosa cell. On the other hand, follicular stimulating hormone (FSH) bind FSH receptor in granulosa cell and stimulate adenylate cyclase via similar way like in theca cell. Granulosa cell converts androgen into estrogen by CYP19A1. In PCOS, hyperplasia of the theca interna is frequently observed [19]. In addition, various androgen and pro-androgens including testosterone, androstenedione and dehydroepiandrosterone sulfate (DHEAS) as well as the gene expressions related to androgen production, such as CYP17, CYP11A, 3 $\beta$-HSD and LH receptor are elevated in hyperandrogenic women of PCOS $[20,21]$. Nelson et al. [22] demonstrated that androgen production were markedly increased in cultured human theca cells obtained from subjects with PCOS. These observations demonstrate that intrinsic steroidogenic dysregulation in ovary contribute to the increase of androgen in PCOS development.

Second, the pulse frequency of the GnRH in hypothalamus increases and leads to HA in PCOS [1,23]. In healthy women, negative feedback on GnRH occurs by progesterone that is produced in luteal phase. However, HA reduces the negative feedback by progesterone on $\mathrm{GnRH}$, which increases the pulse frequency of GnRH $[23,24]$. These changes lead to FSH deficiency and LH hypersecretion, an increased LH/FSH ratio in the patients with PCOS. This induces increased testosterone production in theca cells.

Third, PCOS presents higher activity of $5 \alpha$-reductase $(5 \alpha R)$. Dihydrotestosterone (DHT) is an endogenous androgen hormone and biologically important for sexual differentiation and maturation of male genitalia. It is the primary androgen in the genitalia, prostate gland, skin and hair follicles [25]. It has 2 to 3 fold higher affinity for androgen receptor (AR) and slower dissociation rate from $A R$ than testosterone. The enzyme $5 \alpha R$ 
converts testosterone to DHT that is more potent androgen. The overexpression of $5 \alpha R$ activity is associated with hirsutism, androgenic alopecia and acne [26]. Increased $5 \alpha$ R activity was observed in granulosa cells and cultured fibroblasts that were obtained from the patients with PCOS $[27,28]$. These studies suggest the important role of increased $5 \alpha$ R activity in HA.

Fourth, there are several evidences that the AR plays an important role in the pathogenesis of PCOS [29-31]. The hormonal activities of androgens is mediated in target cells by binding AR. The mouse models with genetically inactivated AR showed similar phenotypes resembling those in human PCOS [32-35]. Clinically, an antagonist of the AR, flutamide recovers regular menstruation and ovulation. The AR antagonist restores the decreased sensitivity of the GnRH pulse generator by estrogen and progesterone $[36,37]$. It improves hirsutism and acne in PCOS patients [38-40]. The human $A R$ gene contains a CAG repeats region in it's $\mathrm{N}$ terminal transcription activation region. Several researches examined the association between the number of CAG repeats and activity of AR in PCOS [41]. Some studies also evaluate the relationship between CAG repeat length and intrafollicular hormonal changes $[42,43]$. Although the clinical significance of this polymorphic site is not determined yet, these observations suggest the importance of $A R$ in the pathogenesis of PCOS [44-46].

Fifth, prenatal hyperandrogenic environment might be associated with PCOS development. Well established PCOS animal models suggest a crucial role of androgen exposure during prenatal and early postnatal period. Animal studies have demonstrated that excess androgen exposure during critical developmental windows leads to phenotypes resembling human PCOS in offsprings, such as ovulatory dysfunction in early reproductive period, polycystic ovaries, hyperandrogenemia and LH hypersecretion [47-49]. The effects of maternal circulation androgen levels on ovarian function in adolescence have been investigated. The study showed that maternal circulating total testosterone levels at 18 gestational weeks were significantly associated with early follicular phase circulating anti-mullerian hormone (AMH) levels in female adolescent offspring. On the other hand, early postnatal testosterone treatment does not induce the classical PCOS phenotypes which are observed by prenatal exposure to excess testosterone in animal model. The timing of exposure to androgen during development is important for the pathogenesis of PCOS.

$\mathrm{HA}$ is considered to be both a cause and a consequence of PCOS and traps the patient in a vicious cycle.

\section{Insulin Resistance}

Epidemiological studies have demonstrated a clear association between PCOS and obesity. The majority of women with PCOS is either obese or overweight [50]. Moderate weight loss of $5 \%$ body weight results in reduction in insulin concentrations and clinical improvement in recovering regular menstrual cycles and a reduction in hirsutism [51]. On the other hand, 47\% of lean women with PCOS showed IR [52]. These observations suggest the importance of insulin sensitivity in the pathogenesis of PCOS. Although PCO and obesity have a synergistic deleterious effect on glucose tolerance, IR is independent of obesity, changes in body composition and impairment of glucose tolerance [53]. It is estimated that 50\% to $90 \%$ of women who diagnosed with PCOS have IR [54]. HA aggravate IR by reducing the sensitivity and expression level of glucose transporter protein-4 (Glu-4), inhibiting the degradation of insulin by the liver, and exacerbating central obesity. On the other hand, IR exacerbates HA through several pathways and play a critical role in developing PCOS.

Insulin stimulates the production of androgens in PCOS. Adipose tissue and muscle become IR due to HA in PCOS. Hyperinsulinemia develops as a compensatory response to IR in PCOS. However, theca cells remain insulin sensitive. Insulin interacts synergistically with $\mathrm{LH}$ and stimulates the androgen production in theca cells [55-57]. In addition, the steroidogenic enzyme expression, especially CYP17 is increased in response to hyperinsulinemia and more androgens are produced in theca cell [58]. The insulin also stimulates adrenal androgen production $[59,60]$. The exact mechanisms how insulin regulate adrenal androgen production has not been elucidated yet. Possible explanation includes dysregulation of steroid synthesis enzyme $11 \beta-H S D$ and hyperstimulation of CYP17 activity in the adrenal gland $[61,62]$.

Insulin inhibits the production of sex hormone-binding globulin (SHBG) in hepatocytes. SHBG is a homodimeric plasma glycoprotein, which is produced by hepatocytes [63]. SHBG transports sex hormones by binding to circulating sex steroids with high affinity. The testosterone binded to SHBG does not have biological effects and 1\% to $2 \%$ of unbound free testosterone shows biological activity. Therefore, SHBG regulates the concentration of bioactive sex hormones $[63,64]$. The SHBG production is regulated by endocrine and metabolic factors [65]. Two related transcription factors, HNF- $4 \alpha$ and COUP-TF1 compete for the same DNA-binding site close to the SHBG transcription start site [66]. The binding of HNF-4 $\alpha$ to the transcription factor-binding site increases SHBG promoter activity while the 
binding of COUP-TF1 represses it [63]. Insulin does not directly regulate $\mathrm{SHBG}$ gene transcription. The mechanisms in which insulin represses hepatic SHBG production has not been elucidated yet, it has been reported that metabolic change including hyperinsulinemia reduces HNF- $4 \alpha$ mRNA and protein levels in the liver [67]. If HNF-4 $\alpha$ level is decreased and COUP-TF1 binds at the transcription site, and SHBG transcription is blocked [64]. Thereby plasma free testosterone levels raise and IR aggravates HA.

In addition, the $5 \alpha R$ activity is closely associated with the body mass index and fasting insulin levels in women with PCOS [46].

\section{Neuroendocrine Abnormality: An Attention Grabbing Pathogenesis of Polycystic Ovarian Syndrome}

Animal models of PCOS shed light on the critical role of extraovarian mechanism in developing PCOS. Caldwell et al. [68] combined a mouse model of DHT-induced PCOS with global and neuron - and granulosa cell specific AR knock out (ARKO) mice to investigate which sites of androgen action is critical in the development PCOS. The authors found that ovariectomized ARKO hosts recovered normal estrous cycles and follicular development when those were transplanted with wild type ovary despite DHT treatment. Additionally, female mice lacking AR actions in the brain were protected against metabolic features of DHT induced PCOS traits. This suggests that extraovarian AR, brain is key loci for androgen action in generating PCOS and neuroendocrine androgen driven mechanism is a critical pathway in the development PCOS. In terms of neuroendocrine abnormalities, several pathways have been elucidated as follows.

\section{Neuroendocrine Abnormalities of Hypothalamus Pituitary Adrenal Axis, in PCOS}

HA and IR cause neuroendocrine abnormalities of adrenal glands as well in PCOS. The adult adrenal gland consists of cortex and medulla [69]. The adrenal cortex is divided into three histologic and functional zone as follows: from outer to inner, zona glomerulosa, zona fasciculata, and zona reticularis. Zona reticularis produces adrenal androgen and small amount of cortisol. Adrenal gland secrets androgen in response to adrenocortical hormone (ACTH), which is a 39-amino acid peptide synthesized and secreted by the anterior pituitary gland. Adrenal dehydroepiandrosterone (DHEA) is converted to DHEAS by sulfotransfer- ase $2 \mathrm{~A} 1$ primarily in several organs including adrenal gland. The enzyme expression in the ovary is limited. Therefore, the source of circulating DHEAS is the adrenal gland and the adrenal HA is defined by increased serum DHEAS level [70].

The greater response of adrenal gland to ACTH is a common feature of patients with PCOS and adrenal HA. Compared with PCOS patients without adrenal HA, PCOS patients with adrenal HA present higher reactivity of adrenal gland to ACTH [71]. The oxidoreductase $11 \beta$-HSD oxidize cortisol to inactive cortisone. The dysregulation of $11 \beta$-HSD leads to increased metabolic clearance of cortisol, which leads to compensatory rise in ACTH secretion to maintain normal cortisol level. This compensatory response increases adrenal production of androgens [62]. Additionally, prenatal androgen exposure leads to PCOS in the offspring. Zhou et al. [72] demonstrated that prenatal exposure to androgen excess induces irreversible changes in adrenal cortex and lead to hypersecretion of adrenal androgen excess in adulthood using female rhesus monkey. With prenatally androgenized female rhesus monkey model, life style intervention preventing increased adiposity in adolescent reduced the risk of acquiring PCOS related metabolic abnormalities in adulthood [73]. This observation suggested hyperinsulinemia and increased adiposity as potential mediators for adrenal HA. This is supported by the observation that insulin sensitizer diminishes DHEAS response to ACTH stimulation [74]. Aromatase activity in human placenta prevents adverse effect of maternal hyperandrogenemia on the fetus. Therefore, inherent maternal steroidogenic defect may lead to the development of adrenal HA in PCOS [70].

\section{Neuroendocrine Abnormalities of Hypothalamus Pituitary Ovarian Axis in PCOS}

Previously, we mentioned about the imbalance of hypothalamic pituitary ovarian axis regulation briefly. Increased LH pulse frequency and an increase in LH to FSH ratio are frequently observed in patients with PCOS. The cause of this observation is the LH hypersecretion by an increased pulse frequency stimulation of $\mathrm{GnRH}$ and exaggerated $\mathrm{LH}$ response to $\mathrm{GnRH}$, the key endocrine defect of the hypothalamus-pituitary-ovarian axis in PCOS [75]. Increased GnRH pulse frequency and LH secretion in PCOS is less responsive to exogenous estrogen and progesterone. Impaired negative feedbacks of estradiol and progesterone have been presented in prenatal testosterone-treated animal models $[76,77]$. This reflect impaired ovarian steroids negative feedback to $\mathrm{GnRH}$ neurons $[23,78]$. High concentrations of ovarian steroids are required to reduce pulsatile LH secretion and sensitivity 
to steroid hormone can be restored with AR antagonist $[37,78]$. The altered estrous cycles and GABAergic drive to $\mathrm{GnRH}$ neurons were recovered by flutamide, $A R$ antagonist in prenatally androgenized mice [79]. Prenatal DHT treatment in brain-specific ARKO mouse did not present PCOS features, which supports the neuroendocrine role androgens in disturbing the HPO axis [68]. These findings provide evidence that negative feedback to $\mathrm{GnRH}$ neuron is mediated directly via the AR. Recent evidence demonstrates the role of $\mathrm{HA}$ in disrupting the neuroendocrine circuit of hypothalamus-pituitary axis [80]. Recently, two arcuate nucleus populations have been getting attention, the arcuate nucleus GABAergic neurons and the kisspeptin-/neurokinin B-/dynorphin-expressing 'KNDy (kisspeptin, neurokinin B and dynorphin)' neurons. The ewes which prenatally treated with testosterone present an imbalance in the KNDy neurons within the arcuate nucleus reflecting reduced inhibitory neuropeptide without changing in stimulatory neuropeptides [81].

\section{Neuroendocrine Abnormalities of Hypothalamus Pituitary Adipose Axis in PCOS}

Adipose dysfunction has been suggested as a potential contributor for PCOS development in androgen-excess PCOS animal models. Adipocytes secrete high amounts of fatty acid (FA) and adipokines that induce IR, which in turn results in ectopic accumulation of lipids in various organs such as liver and skeletal muscle [82]. Obesity induces adipocytes to become hypertrophic. Non-adipose tissues become resistant to leptin (a family member of adipokine) and insulin action. Adiponectin is a protein hormone which is produced in adipose tissue. The hormone is involved in regulating glucose level, oxidizing FA and improving IR.

Several studies have demonstrated the association between androgens and adipose tissue function in developing PCOS. Androgenized female mice presented increased adiposity and adipocyte hypertrophy [83]. Intra-abdominal fat storage is positively correlated with serum androgen levels in normal weight PCOS women [84]. The PCOS animal model using Rhesus monkey shows impaired adult preadipocyte differentiation in abdominal adipose [85]. In addition, in PCOS sheep model, morphology of adipocyte changes before insulin insensitivity and adiposity become evident [86]. These observations suggest adipocyte dysfunction may precede the onset of metabolic dysfunction.

Adiponectin is decreased in PCOS patients [87]. Hyperinsulinemia and IR which are observed in metabolic diseases have an association with hypoadiponectinemia. Hypoadiponectinemia affects insulin and gonadotropin action, which can influence ovarian function indirectly [88]. Neuron specific ARKO female mice present some PCOS metabolic features including hyperglycemia, adipocyte hypertrophy, and decreased serum adiponectin levels while global ARKO mice are protected against metabolic dysfunction [68]. Comparing the classic DHT-induced PCOS mouse model, adiponectin knockouts mice show more IR. However, the mice overexpressing adiponectin from adipose tissue remained metabolically healthy under DHT exposure [89]. This study demonstrates the importance of the role adiponectin for the development of PCOS.

The role of adipokines has been proposed for underlying pathogenesis of PCOS $[90,91]$. Androgen excess is associated with visceral adiposity. In a PCOS mouse model, DHT induced leptin failure with decreased energy expenditure, which promotes visceral adiposity. This metabolic change associated with reduced proopiomelanocortin neuronal innervation in the dorsomedial hypothalamus [83]. These result suggested the importance of androgen-brain-adipocyte axis and the adipose tissue function in PCOS development.

\section{Current Research in Genetic Basis of PCOS}

Although PCOS is considered a complex endocrine disease with multifactorial etiology. Number of studies have demonstrated the importance of genetic basis in the development of PCOS [92-95]. In 1968, Cooper et al. [95] analyzed 18 families who were diagnosed with Stein-Levanthal syndrome and concluded that the disease has an hereditary basis with hereditary transmission potential.

In addition to familial clustering of cases, a greater concordance of the syndrome in identical than in non-identical twin pairs provided strong evidence for a genetic basis of the syndrome. Vink et al. [93] examined the contributions of genetic factors in a Dutch twin family study. The authors used the Netherlands Twin Register Data which was consisted of 1,332 monozygotic (MZ) twins and 1,873 dizygotic (DZ) twins. The 2003 Rotterdam consensus criteria were adopted for the diagnosis of PCOS. The prevalence of PCOS did not differ among groups, DZ twin, MZ twin and singleton sisters. However, the MZ correlations were twice as high as the DZ twins and sister correlations, suggesting genetic influence of on PCOS development.

Legro et al. [92] investigated 115 sisters of 80 probands with PCOS from unrelated families. The sisters were compared with 70 healthy control women with regular menses, normal glucose 
tolerance and no evidence of hyperandrogenemia. The familial aggregation of hyperandrogenemia was found in PCOS relatives, which suggests a genetic traits of endocrinopathy.

Association studies have been used to identify disease susceptibility loci in genetic studies. Most of genetic research has been performed with genetic association study to identify PCOS risk genes. These studies focus on candidate genes of interest which were selected by suggested pathogenesis of the disease. The genetic association study has several advantages. It requires relatively small sample sizes and less costs. However, this approach has some limitations on complex disease including PCOS, diabetes mellitus, and hypertension because of lack of understanding of pathophysiology of these complicated diseases and gene function. Genetic association studies to elucidate the role of genetics in PCOS development have been performed on more than dozens of candidate genes that are involved in the potential pathogenesis of PCOS, such as IR and hyperandrogenemia. The attempts have been relatively unsuccessful so far. There are several reasons. One major problem remains the inconclusive definition of the PCOS phenotype. Another problem is in the underlying complexity of the disorder. The metabolic feature of PCOS with combined dysregulation of abnormal steroidogenesis, fat metabolism and glucose metabolism has led to the suggestion of numerous candidate genes. Lastly, environmental factors play an important role in developing PCOS.

Genome wide association study (GWAS) compares common genetic variants in large numbers of affected cases to those in unaffected controls to determine whether a significant association exist between genetic variant and disease. GWAS provides greater power than genetic linkage studies to identify small to modest effect.

In 2010, the first GWAS was performed to identify genetic markers for PCOS in a Han Chinese population [96]. The authors examined more than 3,000 PCOS patients and 6,000 controls for the analysis. PCOS were diagnosed according to the revised 2003 Rotterdam consensus criteria. Three susceptibility loci (chr2p16.3, rs13405728; chr2p21, rs13429458; chr9q33.3, rs2479106) for PCOS in Han Chinese population. Several genes that located near these loci including $L H C G R, F S H R, T H A D A$, and DENND1A were provided as potential candidate genes for PCOS.

The researchers conducted further GWAS study to explore additional risk regions for PCOS by analyzing additional 1510 PCOS cases and 2106 controls [97]. Eight new PCOS association loci (chr9q22.32, rs4385527; chr9q22.32, rs3802457; chr11q22.1, rs 1894116; chr12q13.2, rs705702; chr12q14.3, rs2272046; chr16q142.1, rs4784165; chr19p13.3, rs2059807; chr20q13.2, rs6022786) were identified. The candidate genes at the associated loci were follows: C9orf3, YAP1, RAB5B, SUOX, HMGA2, TOX3, INSR, SUMO1p1. Those genes were associated with IR, sexual hormones and organ growth.

The prevalence of genetic polymorphisms differs among ethnic groups. Previous GWAS results suggest 2p16.3 encoding LHCGR and FSHR genes as a PCOS susceptibility locus in Han Chinese population. Mutharasan et al. [98] tested the association between PCOS and 96 SNPs mapping to the genomic region encompassing LHCGR and FSHR in US Caucasian population, which consists of 905 PCOS cases and 956 control cases. The 2 p16.3 locus, rs 13405728 did not show significant association with PCOS in US Caucasian, which was susceptibility locus in Han Chinese population. However, rs 10495960 and rs1922476 on 2p16.3 were strongly associated with PCOS in the study. These loci were mapped to FSHR and LHCGR, respectively. Therefore, FSHR and LHCGR gene were provided as potential candidate genes in PCOS pathogenesis.

Another GWAS study with largest cohort was conducted in 2015 [99]. Self-reported 5,184 PCOS cases and 82,759 controls studies of the Caucasia European ancestors were included. The authors presented follow up in additional studies in clinically confirmed cases because of the limitation for the diagnosis of PCOS by including self-reported PCOS cases. Six loci including rs1351592, rs 11225154, rs7563201, rs11031006, rs13164856, rs1275468 showed a significant association with PCOS in the meta-analysis and follow up studies. Those novel loci were located near by genes ERBB4, YAP1, THADA, FSHB, RAD50, KRR1, respectively. $E R B B 4$ encodes a member of epidermal growth factor receptor (EGFR) family. EGFR signaling mediates LH induced steroidogenesis, which is involved in late follicular maturation. In addition, the researchers find a consistent association between higher serum AMH concentrations and PCOS susceptibility allele.

To identify susceptibility loci for the HA and ovulation, a discovery GWAS study was carried out with 2,783 NIH phenotype of PCOS cases and 4,195 control women in European ancestry population [100]. Three loci including two novel loci mapping to chr8p23.1 GATA4/NEIL2 and chr11p14.1 FSHB/ARL14EP and one previously reported locus chr9q22.32 C9orf3/FANCC show significant associations with PCOS phenotype. Of those loci, chr11p14.1 rs11031006 is in the FSHB gene. With this result, the authors suggested the crucial role of gonadotropin in PCOS pathogenesis. The chr9q22.32 PCOS locus was common in the Chinese and European cohorts. The authors suggested that chr9q22.32 region presents an evolutionary genetic susceptibil- 
ity factor for PCOS.

Current understanding of the role of genetics in PCOS development is that genetic factors affect ovarian androgenic activity and metabolic dysfunction and environmental circumstances aggravate HA and IR in PCOS.

\section{Conclusion}

PCOS is one of the most common endocrine disorder in reproductive age women. The clinical symptom varies including oligo-ovulation or anovulation, HA and PCOM. In addition, the endocrinopathy lead to the adverse cardiovascular outcome, increased risk of metabolic syndrome and diabetes and subfertility. Life style modification and oral contraceptives may help with improving the clinical symptom of PCOS. HA and IR play a irreplaceable role in developing PCOS. In addition to HA and $I R$, hormonal, endocrinal and reproductive dysfunction that is found in PCOS include LH hypersecretion, ovulatory disturbance, aberrant follicular maturation, chronic inflammation and oxidative stress. The alteration of factors unrelated to oocyte and embryo morphology may reduce the competence of maturating oocytes through endocrine and local paracrine/autocrine actions, resulting in a lower pregnancy rate in women with PCOS. Genetic factors affect ovarian androgenic activity and metabolic dysfunction observed in PCOS. Additionally, environmental factors aggravates HA and IR and lead to the development of PCOS in individual who has genetic susceptibility to PCOS. However, there has been no cure for PCOS. The etiology of this complex condition still remains obscure. That would be the reason that we have not found the cure for the PCOS. Recent researches have focused on elucidating the role of novel genetic mechanisms including epigenetics and microRNA. Further understanding on these will help to provide cure for the patients who suffer from the endocrinopathy.

\section{Acknowledgements}

This research was supported by the National Research Foundation of Korea (NRF) grant funded by the Korea government (MSIT) (No. NRF-2019R1G1A1011557).

\section{References}

1. Azziz R, Carmina E, Chen Z, Dunaif A, Laven JS, Legro RS, et al. Polycystic ovary syndrome. Nat Rev Dis Primers 2016;2:16057.

2. Goodarzi MO, Dumesic DA, Chazenbalk G, Azziz R. Polycystic ovary syndrome: etiology, pathogenesis and diagnosis. Nat Rev Endocrinol 2011;7:219-31.

3. Conway G, Dewailly D, Diamanti-Kandarakis E, Escobar-Morreale HF, Franks S, Gambineri A, et al.; ESE PCOS Special Interest Group. The polycystic ovary syndrome: a position statement from the European Society of Endocrinology. Eur J Endocrinol 2014;171:P1-29.

4. Stein IF, Leventhal ML. Amenorrhea associated with bilateral polycystic ovaries. Am J Obstet Gynecol 1935;29:181-91.

5. Zawadzki JK. Diagnostic criteria for polycystic ovary syndrome (a rational approach). Polycystic Ovary Syndrome 1992:377-84.

6. Teede HJ, Misso ML, Costello MF, Dokras A, Laven J, Moran L, et al.; International PCOS Network. Recommendations from the international evidence-based guideline for the assessment and management of polycystic ovary syndrome. Fertil Steril 2018;110:364-79.

7. Escobar-Morreale HF. Polycystic ovary syndrome: definition, aetiology, diagnosis and treatment. Nat Rev Endocrinol 2018;14:270-84.

8. DeUgarte CM, Bartolucci AA, Azziz R. Prevalence of insulin resistance in the polycystic ovary syndrome using the homeostasis model assessment. Fertil Steril 2005;83:1454-60.

9. Dunaif A, Wu X, Lee A, Diamanti-Kandarakis E. Defects in insulin receptor signaling in vivo in the polycystic ovary syndrome (PCOS). Am J Physiol Endocrinol Metab 2001;281:E392-9.

10. Sengoku K, Tamate K, Takuma N, Yoshida T, Goishi K, Ishikawa M. The chromosomal normality of unfertilized oocytes from patients with polycystic ovarian syndrome. Hum Reprod 1997;12:474-7.

11. Ludwig M, Finas DF, al-Hasani $S$, Diedrich $K$, Ortmann O. Oocyte quality and treatment outcome in intracytoplasmic sperm injection cycles of polycystic ovarian syndrome patients. Hum Reprod 1999;14:354-8.

12. Mulders AG, Laven JS, Imani B, Eijkemans MJ, Fauser BC. IVF outcome in anovulatory infertility (WHO group 2)--including polycystic ovary syndrome--following previous unsuccessful ovulation induction. Reprod Biomed Online 2003;7:50-8.

13. Heijnen EM, Eijkemans MJ, Hughes EG, Laven JS, Macklon NS, Fauser BC. A meta-analysis of outcomes of conventional IVF in women with polycystic ovary syndrome. Hum Reprod Update 2006;12:13-21.

14. Weghofer A, Munne S, Chen S, Barad D, Gleicher N. Lack of association between polycystic ovary syndrome and embryonic aneuploidy. Fertil Steril 2007;88:900-5.

15. Sahu B, Ozturk O, Ranierri M, Serhal P. Comparison of oocyte quality and intracytoplasmic sperm injection outcome in women with isolated polycystic ovaries or polycystic ovarian syndrome. Arch Gynecol Obstet 2008;277:239-44.

16. Boomsma CM, Fauser BC, Macklon NS. Pregnancy complications in women with polycystic ovary syndrome. Semin Reprod Med 2008;26:72-84. 
17. Qiao J, Feng HL. Extra- and intra-ovarian factors in polycystic ovary syndrome: impact on oocyte maturation and embryo developmental competence. Hum Reprod Update 2011;17:17-33.

18. Erickson GF, Magoffin DA, Dyer CA, Hofeditz C. The ovarian androgen producing cells: a review of structure/function relationships. Endocr Rev 1985;6:371-99.

19. Mahajan DK. Steroidogenesis in human polycystic ovary. Endocrinol Metab Clin North Am 1988;17:751-69.

20. Keefe CC, Goldman MM, Zhang K, Clarke N, Reitz RE, Welt CK. Simultaneous measurement of thirteen steroid hormones in women with polycystic ovary syndrome and control women using liquid chromatography-tandem mass spectrometry. PLoS One 2014;9:e93805.

21. Palomba S, Falbo A, Chiossi G, Muscogiuri G, Fornaciari E, Orio F, et al. Lipid profile in nonobese pregnant women with polycystic ovary syndrome: a prospective controlled clinical study. Steroids 2014;88:3643.

22. Nelson VL, Legro RS, Strauss JF 3rd, McAllister JM. Augmented androgen production is a stable steroidogenic phenotype of propagated theca cells from polycystic ovaries. Mol Endocrinol 1999;13:946-57.

23. Burt Solorzano CM, Beller JP, Abshire MY, Collins JS, McCartney CR, Marshall JC. Neuroendocrine dysfunction in polycystic ovary syndrome. Steroids 2012;77:332-7.

24. Rojas J, Chávez M, Olivar L, Rojas M, Morillo J, Mejias J, et al. Polycystic ovary syndrome, insulin resistance, and obesity: navigating the pathophysiologic labyrinth. Int J Reprod Med 2014;2014:719050.

25. Marks LS. 5alpha-reductase: history and clinical importance. Rev Urol 2004;6 Suppl 9(Suppl 9):S11-21.

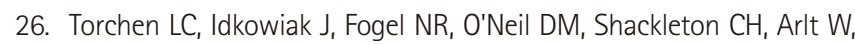
et al. Evidence for increased $5 \alpha$-reductase activity during early childhood in daughters of women with polycystic ovary syndrome. J Clin Endocrinol Metab 2016;101:2069-75.

27. Boda D, Păun D, Diaconeasa A. Evaluation of 5-alpha reductase activity on cultured fibroblast in patients with hyperandrogenemia. Rom J Intern Med 2009;47:67-73.

28. Magoffin DA. Ovarian enzyme activities in women with polycystic ovary syndrome. Fertil Steril 2006;86 Suppl 1:S9-11.

29. Walters KA, Handelsman DJ. Role of androgens in the ovary. Mol Cell Endocrinol 2018;465:36-47.

30. Brock O, De Mees C, Bakker J. Hypothalamic expression of oestrogen receptor $\alpha$ and androgen receptor is sex-, age- and region-dependent in mice. J Neuroendocrinol 2015;27:264-76.

31. Mogi $K$, Takanashi H, Nagasawa M, Kikusui T. Sex differences in spatiotemporal expression of $A R, E R \alpha$, and $E R \beta$ mRNA in the perinatal mouse brain. Neurosci Lett 2015;584:88-92.

32. Hu YC, Wang PH, Yeh $S$, Wang RS, Xie $C, X u$ O, et al. Subfertility and defective folliculogenesis in female mice lacking androgen receptor.
Proc Natl Acad Sci U S A 2004;101:11209-14.

33. Ma $Y$, Andrisse $S$, Chen $Y$, Childress $S, X u e ~ P$, Wang $Z$, et al. Androgen receptor in the ovary theca cells plays a critical role in androgeninduced reproductive dysfunction. Endocrinology 2017;158:98-108.

34. Shiina $H_{1}$ Matsumoto $T$, Sato $T$, Igarashi $K_{1}$ Miyamoto J, Takemasa $S$, et al. Premature ovarian failure in androgen receptor-deficient mice. Proc Natl Acad Sci U S A 2006;103:224-9.

35. Yeh S, Tsai MY, Xu O, Mu XM, Lardy H, Huang KE, et al. Generation and characterization of androgen receptor knockout (ARKO) mice: an in vivo model for the study of androgen functions in selective tissues. Proc Natl Acad Sci U S A 2002;99:13498-503.

36. Paradisi $R$, Fabbri $R$, Battaglia $C$, Venturoli $S$. Ovulatory effects of flutamide in the polycystic ovary syndrome. Gynecol Endocrinol 2013;29:391-5.

37. Eagleson CA, Gingrich MB, Pastor CL, Arora TK, Burt CM, Evans WS, et al. Polycystic ovarian syndrome: evidence that flutamide restores sensitivity of the gonadotropin-releasing hormone pulse generator to inhibition by estradiol and progesterone. J Clin Endocrinol Metab 2000;85:4047-52.

38. Moghetti P, Tosi F, Tosti A, Negri C, Misciali C, Perrone F, et al. Comparison of spironolactone, flutamide, and finasteride efficacy in the treatment of hirsutism: a randomized, double blind, placebocontrolled trial. J Clin Endocrinol Metab 2000;85:89-94.

39. Venturoli S, Marescalchi O, Colombo FM, Macrelli S, Ravaioli B, Bagnoli $A$, et al. A prospective randomized trial comparing low dose flutamide, finasteride, ketoconazole, and cyproterone acetate-estrogen regimens in the treatment of hirsutism. J Clin Endocrinol Metab 1999:84:1304-10.

40. Calaf J, López E, Millet A, Alcañiz J, Fortuny A, Vidal O, et al.; Spanish Working Group for Hirsutism. Long-term efficacy and tolerability of flutamide combined with oral contraception in moderate to severe hirsutism: a 12-month, double-blind, parallel clinical trial. J Clin Endocrinol Metab 2007;92:3446-52.

41. Baculescu N. The role of androgen receptor activity mediated by the CAG repeat polymorphism in the pathogenesis of PCOS. J Med Life 2013;6:18-25.

42. Borgbo T, Macek M Sr, Chrudimska J, Jeppesen JV, Hansen LL, Andersen CY. Size matters: associations between the androgen receptor CAG repeat length and the intrafollicular hormone milieu. Mol Cell Endocrinol 2016;419:12-7.

43. Skrgatic L, Baldani DP, Cerne JZ, Ferk P, Gersak K. CAG repeat polymorphism in androgen receptor gene is not directly associated with polycystic ovary syndrome but influences serum testosterone levels. J Steroid Biochem Mol Biol 2012;128:107-12.

44. Jakimiuk $A$, Weitsman SR, Magoffin DA. 5alpha-reductase activity in women with polycystic ovary syndrome. J Clin Endocrinol Metab 
1999;84:2414-8.

45. Skałba P, Dabkowska-Huć A, Kazimierczak W, Samojedny A, Samojedny MP, Chełmicki Z. Content of 5-alpha-reductase (type 1 and type 2) mRNA in dermal papillae from the lower abdominal region in women with hirsutism. Clin Exp Dermatol 2006;31:564-70.

46. Vassiliadi DA, Barber TM, Hughes BA, McCarthy MI, Wass JA, Franks $S$, et al. Increased 5 alpha-reductase activity and adrenocortical drive in women with polycystic ovary syndrome. J Clin Endocrinol Metab 2009;94:3558-66.

47. Walters KA. Role of androgens in normal and pathological ovarian function. Reproduction 2015;149:R193-218.

48. Padmanabhan $V$, Veiga-Lopez A. Sheep models of polycystic ovary syndrome phenotype. Mol Cell Endocrinol 2013;373:8-20.

49. Abbott DH, Nicol LE, Levine JE, Xu N, Goodarzi MO, Dumesic DA. Nonhuman primate models of polycystic ovary syndrome. Mol Cell Endocrinol 2013;373:21-8.

50. Balen AH, Conway GS, Kaltsas G, Techatrasak K, Manning PJ, West C, et al. Polycystic ovary syndrome: the spectrum of the disorder in 1741 patients. Hum Reprod 1995;10:2107-11.

51. Kiddy DS, Hamilton-Fairley D, Bush A, Short F, Anyaoku V, Reed MJ, et al. Improvement in endocrine and ovarian function during dietary treatment of obese women with polycystic ovary syndrome. Clin Endocrinol (Oxf) 1992;36:105-11.

52. Yildizhan B, Anik Ilhan G, Pekin T. The impact of insulin resistance on clinical, hormonal and metabolic parameters in lean women with polycystic ovary syndrome. J Obstet Gynaecol 2016;36:893-6.

53. Dunaif $A$, Segal KR, Futterweit W, Dobrjansky A. Profound peripheral insulin resistance, independent of obesity, in polycystic ovary syndrome. Diabetes 1989;38:1165-74.

54. Venkatesan AM, Dunaif A, Corbould A. Insulin resistance in polycystic ovary syndrome: progress and paradoxes. Recent Prog Horm Res 2001;56:295-308.

55. Bergh C, Carlsson B, Olsson JH, Selleskog U, Hillensjö T. Regulation of androgen production in cultured human thecal cells by insulin-like growth factor I and insulin. Fertil Steril 1993;59:323-31.

56. Cara JF, Rosenfield RL Insulin-like growth factor I and insulin potentiate luteinizing hormone-induced androgen synthesis by rat ovarian thecal-interstitial cells. Endocrinology 1988;123:733-9.

57. Nahum R, Thong KJ, Hillier SG. Metabolic regulation of androgen production by human thecal cells in vitro. Hum Reprod 1995;10:7581.

58. Munir I, Yen HW, Geller DH, Torbati D, Bierden RM, Weitsman SR, et al. Insulin augmentation of 17alpha-hydroxylase activity is mediated by phosphatidyl inositol 3-kinase but not extracellular signalregulated kinase-1/2 in human ovarian theca cells. Endocrinology 2004;145:175-83.
59. Kristiansen SB, Endoh A, Casson PR, Buster JE, Hornsby PJ. Induction of steroidogenic enzyme genes by insulin and IGF-I in cultured adult human adrenocortical cells. Steroids 1997;62:258-65.

60. Rittmaster RS, Deshwal N, Lehman L. The role of adrenal hyperandrogenism, insulin resistance, and obesity in the pathogenesis of polycystic ovarian syndrome. J Clin Endocrinol Metab 1993;76:1295300.

61. Moghetti P, Castello R, Negri C, Tosi F, Spiazzi GG, Brun E, et al. Insulin infusion amplifies 17 alpha-hydroxycorticosteroid intermediates response to adrenocorticotropin in hyperandrogenic women: apparent relative impairment of 17,20-lyase activity. J Clin Endocrinol Metab 1996;81:881-6.

62. Rodin A, Thakkar H, Taylor N, Clayton R. Hyperandrogenism in polycystic ovary syndrome. Evidence of dysregulation of 11 betahydroxysteroid dehydrogenase. N Engl J Med 1994;330:460-5.

63. Hammond GL, Wu TS, Simard M. Evolving utility of sex hormonebinding globulin measurements in clinical medicine. Curr Opin Endocrinol Diabetes Obes 2012;19:183-9.

64. Zhu JL, Chen Z, Feng WJ, Long SL, Mo ZC. Sex hormone-binding globulin and polycystic ovary syndrome. Clin Chim Acta 2019;499:142-8.

65. Hammond GL. Diverse roles for sex hormone-binding globulin in reproduction. Biol Reprod 2011;85:431-41.

66. Jänne M, Hammond GL. Hepatocyte nuclear factor-4 controls transcription from a TATA-less human sex hormone-binding globulin gene promoter. J Biol Chem 1998;273:34105-14.

67. Xie $X$, Liao H, Dang H, Pang W, Guan Y, Wang $X_{1}$ et al. Down-regulation of hepatic HNF4alpha gene expression during hyperinsulinemia via SREBPs. Mol Endocrinol 2009;23:434-43.

68. Caldwell ASL, Edwards MC, Desai $R$, Jimenez $M$, Gilchrist RB, Handelsman DJ, et al. Neuroendocrine androgen action is a key extraovarian mediator in the development of polycystic ovary syndrome. Proc Natl Acad Sci U S A 2017;114:E3334-43.

69. Antoniou-Tsigkos A, Zapanti E, Ghizzoni L, Mastorakos G. Adrenal androgens. In: Feingold KR, Anawalt B, Boyce A, Chrousos G, Dungan K, Grossman A, eds. Endotext. South Dartmouth: MDText.com, Inc., 2000.

70. Luque-Ramirez M, Escobar-Morreale HF. Adrenal hyperandrogenism and polycystic ovary syndrome. Curr Pharm Des 2016;22:5588-602.

71. Moran C, Reyna R, Boots LS, Azziz R. Adrenocortical hyperresponsiveness to corticotropin in polycystic ovary syndrome patients with adrenal androgen excess. Fertil Steril 2004;81:126-31.

72. Zhou R, Bird IM, Dumesic DA, Abbott DH. Adrenal hyperandrogenism is induced by fetal androgen excess in a rhesus monkey model of polycystic ovary syndrome. J Clin Endocrinol Metab 2005;90:6630-7.

73. Abbott DH, Tarantal AF, Dumesic DA. Fetal, infant, adolescent and adult phenotypes of polycystic ovary syndrome in prenatally andro- 
genized female rhesus monkeys. Am J Primatol 2009;71:776-84.

74. Zhou R, Bruns CM, Bird IM, Kemnitz JW, Goodfriend TL, Dumesic DA, et al. Pioglitazone improves insulin action and normalizes menstrual cycles in a majority of prenatally androgenized female rhesus monkeys. Reprod Toxicol 2007;23:438-48.

75. McCartney CR, Eagleson CA, Marshall JC. Regulation of gonadotropin secretion: implications for polycystic ovary syndrome. Semin Reprod Med 2002;20:317-26.

76. Abbott DH, Levine JE, Dumesic DA. Translational insight into polycystic ovary syndrome (PCOS) from female monkeys with PCOS-like traits. Curr Pharm Des 2016;22:5625-33.

77. Moore AM, Prescott M, Marshall CJ, Yip SH, Campbell RE. Enhancement of a robust arcuate GABAergic input to gonadotropin-releasing hormone neurons in a model of polycystic ovarian syndrome. Proc Natl Acad Sci U S A 2015;112:596-601.

78. Pastor CL, Griffin-Korf ML, Aloi JA, Evans WS, Marshall JC. Polycystic ovary syndrome: evidence for reduced sensitivity of the gonadotropin-releasing hormone pulse generator to inhibition by estradiol and progesterone. J Clin Endocrinol Metab 1998;83:582-90.

79. Sullivan SD, Moenter SM. Prenatal androgens alter GABAergic drive to gonadotropin-releasing hormone neurons: implications for a common fertility disorder. Proc Natl Acad Sci U S A 2004;101:7129-34.

80. Moore AM, Campbell RE. The neuroendocrine genesis of polycystic ovary syndrome: a role for arcuate nucleus GABA neurons. J Steroid Biochem Mol Biol 2016;160:106-17.

81. Cheng G, Coolen LM, Padmanabhan V, Goodman RL, Lehman MN. The kisspeptin/neurokinin B/dynorphin (KNDy) cell population of the arcuate nucleus: sex differences and effects of prenatal testosterone in sheep. Endocrinology 2010;151:301-11.

82. Vázquez-Vela ME, Torres N, Tovar AR. White adipose tissue as endocrine organ and its role in obesity. Arch Med Res 2008;39:715-28.

83. Nohara K, Laque A, Allard C, Münzberg H, Mauvais-Jarvis F. Central mechanisms of adiposity in adult female mice with androgen excess. Obesity (Silver Spring) 2014;22:1477-84.

84. Dumesic DA, Akopians AL, Madrigal VK, Ramirez E, Margolis DJ, Sarma MK, et al. Hyperandrogenism accompanies increased intraabdominal fat storage in normal weight polycystic ovary syndrome women. J Clin Endocrinol Metab 2016;101:4178-88.

85. Keller E, Chazenbalk GD, Aguilera P, Madrigal V, Grogan T, Elashoff D, et al. Impaired preadipocyte differentiation into adipocytes in subcutaneous abdominal adipose of PCOS-like female rhesus monkeys. Endocrinology 2014;155:2696-703.

86. Cardoso RC, Veiga-Lopez A, Moeller J, Beckett E, Pease A, Keller E, et al. Developmental programming: impact of gestational steroid and metabolic milieus on adiposity and insulin sensitivity in prenatal testosterone-treated female sheep. Endocrinology 2016;157:522-35.
87. Mannerås-Holm L, Leonhardt $H$, Kullberg J, Jennische $E$, Odén $A$, Holm G, et al. Adipose tissue has aberrant morphology and function in PCOS: enlarged adipocytes and low serum adiponectin, but not circulating sex steroids, are strongly associated with insulin resistance. J Clin Endocrinol Metab 2011;96:E304-11.

88. Puttabyatappa M, Padmanabhan V. Ovarian and extra-ovarian mediators in the development of polycystic ovary syndrome. J Mol Endocrinol 2018;61:R161-84.

89. Benrick $A$, Chanclón $B$, Micallef $P$, Wu Y, Hadi L, Shelton JM, et al. Adiponectin protects against development of metabolic disturbances in a PCOS mouse model. Proc Natl Acad Sci U S A 2017;114:E7187-96.

90. Chen $X$, Jia $X$, Qiao J, Guan $Y$, Kang J. Adipokines in reproductive function: a link between obesity and polycystic ovary syndrome. J Mol Endocrinol 2013;50:R21-37.

91. Barber TM, Franks S. Adipocyte biology in polycystic ovary syndrome. Mol Cell Endocrinol 2013;373:68-76.

92. Legro RS, Driscoll D, Strauss JF 3rd, Fox J, Dunaif A. Evidence for a genetic basis for hyperandrogenemia in polycystic ovary syndrome. Proc Natl Acad Sci U S A 1998;95:14956-60.

93. Vink JM, Sadrzadeh S, Lambalk CB, Boomsma DI. Heritability of polycystic ovary syndrome in a Dutch twin-family study. J Clin Endocrinol Metab 2006;91:2100-4.

94. Kahsar-Miller M, Azziz R. The development of the polycystic ovary syndrome: family history as a risk factor. Trends Endocrinol Metab 1998:9:55-8.

95. Cooper HE, Spellacy WN, Prem KA, Cohen WD. Hereditary factors in the Stein-Leventhal syndrome. Am J Obstet Gynecol 1968;100:371-87.

96. Chen ZJ, Zhao H, He L, Shi Y, Qin Y, Shi Y, et al. Genome-wide association study identifies susceptibility loci for polycystic ovary syndrome on chromosome 2p16.3, 2p21 and 9q33.3. Nat Genet 2011;43:55-9.

97. Shi Y, Zhao H, Shi Y, Cao Y, Yang D, Li Z, et al. Genome-wide association study identifies eight new risk loci for polycystic ovary syndrome. Nat Genet 2012;44:1020-5.

98. Mutharasan $P$, Galdones $E$, Peñalver Bernabé $B$, Garcia OA, Jafari $N$, Shea LD, et al. Evidence for chromosome 2p16.3 polycystic ovary syndrome susceptibility locus in affected women of European ancestry. J Clin Endocrinol Metab 2013;98:E185-90.

99. Day FR, Hinds DA, Tung JY, Stolk L, Styrkarsdottir U, Saxena R, et al. Causal mechanisms and balancing selection inferred from genetic associations with polycystic ovary syndrome. Nat Commun 2015;6:8464.

100. Hayes MG, Urbanek M, Ehrmann DA, Armstrong LL, Lee JY, Sisk $R$, et al.; Reproductive Medicine Network. Genome-wide association of polycystic ovary syndrome implicates alterations in gonadotropin secretion in European ancestry populations. Nat Commun 2015;6:7502. 